\title{
How to develop clinical reasoning in medical students and interns based on illness script theory: An experimental study
}

\author{
Somayeh Delavari ${ }^{1}$, Alireza Monajemi ${ }^{* 2,3} \mathbb{D}$, Hamid Reza Baradaran ${ }^{1,4,5}$, Phyo Kyaw Myint ${ }^{5}$, Minoo Yaghmaei ${ }^{6}$, \\ Seyed Kamran Soltani Arabshahi*1(D)
}

Received: 3 Dec 2018

Published: 20 Feb 2020

\section{Abstract}

Background: Although theory explains the development of illness script, it does not provide answers how medical students develop scripts in their learning. To fill the knowledge gap of developing illness script in medical students and interns, this study aimed to investigate the impact of educational strategies inspired by theory in the development of illness scripts.

Methods: A total of 15 medical students and 12 interns participated in an educational intervention that included theory-driven strategies. To evaluate the impact of this intervention, clinical reasoning problem (CRP) and key features (KF) tests were used for before and after the intervention. In addition to descriptive statistics, the differences in participants' pretest and posttest variables were tested using Wilcoxon. Significance level was set at $\mathrm{p} \leq 0.05$ for all tests.

Results: Interns significantly recognized more KF in the posttest. However, no significant difference was found between the pretest and posttest scores in total diagnostic accuracy $(5.41 \pm 1.16$ vs $4.91 \pm 1.44 ; \mathrm{p}=0.111)$ and total correct discriminating score $(0.41 \pm 0.66 \mathrm{vs}$ $1.41 \pm 2.06 ; \mathrm{p}=0.146$ ). Medical students produced less total key features in the posttest, indicating that they became less elaborate in their case processing. However, no significant difference was observed in common KF score $(0.4$ [0.25-0.78] vs 0.9 [0.6-1]; $p=0.791)$ and discriminative key features score $(0.33[0.16-0.33]$ vs $0.22[0.11-0.44] ; \mathrm{p}=0.972)$ in the posttest compared to the pretest.

Conclusion: This study showed that theory-driven educational strategies have an impact on illness script development specifically in interns. It is recommended that this intervention would be tested on those in higher levels of expertise (ie, residents).

Keywords: Illness script, Clinical reasoning, Clinical decision-making, Medical education, Medical student, Education, Undergraduate

Conflicts of Interest: None declared

Funding: Iran University of Medical Sciences

\section{*This work has been published under CC BY-NC-SA 1.0 license. Copyright $\odot$ Iran University of Medical Sciences}

Cite this article as: Delavari S, Monajemi A, Baradaran HR, Myint PK, Yaghmaei M, Soltani Arabshahi K. How to develop clinical reasoning in medical students and interns based on illness script theory: An experimental study. Med J Islam Repub Iran. 2020 (20 Feb);34:9. https://doi.org/10.47176/mjiri.34.9

\section{Introduction}

A 32-year-old woman in the second trimester of pregnancy was brought into the emergency unit because of

Corresponding author: Dr Alireza Monajemi, monajemi@ihcs.ac.ir DrKamran Soltani Arabshahi, soltarab@iums.ac.ir

1. Center for Educational Research in Medical Sciences (CERMS), Department of Medical Education, School of Medicine, Iran University of Medical Sciences, Tehran, Iran

2. Department of Philosophy of Science, Institute for Humanities and Cultural Studies, Tehran, Iran

3. Medical Humanities Department, Virtual University of Medical Sciences, Tehran, Iran

4. Endocrine Research Center, Institute of Endocrinology and Metabolism, Iran University of Medical Sciences, Tehran, Iran

5. Aging Clinical \& Experimental Research Team, Institute of Applied Health Sciences, University of Aberdeen, Aberdeen, UK

6. Department of Obstetrics and Gynecology, School of Medicine, Shahid Beheshti University of Medical Sciences, Tehran, Iran convulsion and deep coma. Her blood pressure was 180/120 mm Hg, temperature $37^{\circ} \mathrm{C}$, and pulse rate of 120

$\uparrow$ What is "already known" in this topic:

Theory-based studies have shown to bring about fresh and insightful ideas for teaching clinical reasoning and may fill in the gap between theory and practice in medical education research.

$\rightarrow$ What this article adds:

Educational strategies inspired by theory are useful in the development of illness scripts of medical interns and clinical reasoning is a learnable skill. 
per minute. There was no previous history of convulsion and hypertension, but she had experienced severe headache since the previous night. A medical doctor examined the patient and diagnosed her with eclampsia because she recognized a pattern: hypertension in the second half of pregnancy, general edema, headache, deep coma, and convulsion without any previous history. The doctor ordered some tests to confirm the diagnosis.

It is well documented that every physician needs a well-structured pattern illness script to make a diagnosis (1-3). Illness script theory explains not only how medical diagnostic knowledge is structured, stored, and retrieved, but also how it influences the interpretation of clinical situations $(4,5)$. Illness scripts are narrative $(6)$, classified (7), and proprietary medical knowledge structures $(2,4)$ that have been developed through clinical experience and practice (2). This structured knowledge is for disease diagnosis and appropriate patient management $(2,7)$. Each illness script consists of fault (pathophysiological mechanism), consequences (signs and symptoms) (2, 5, 7-9), enabling conditions (predisposing factors, boundary conditions, hereditary factors) $(5,10)$, and patient management (11).

The development of illness script is influenced by the integration of individual knowledge and experience in clinical settings $(1,12,13)$, which is critical for a transition from a novice to an expert $(1,12,14)$. The theoretical process of illness script development includes 4 steps (15, 16). First, the person acquires an elaborated network of knowledge for explaining the causes and consequences of diseases based on underlying pathophysiological mechanisms, which is encapsulated into simplified causal models for explaining signs and symptoms in the second step. In the third step, extensive practice, with real patients leads to emergence of narrative structures called illness script. In the last step, as a result of extensive clinical knowledge and clinical practice, illness scripts are enriched with multiple clinical presentations $(1,15,16)$.

In medical education, it is highly important to find the best method to train medical students to develop their illness scripts. According to a narrative review by Schmidt and Mamede, several studies introduced various methods for clinical reasoning learning (3). However, the most important criticisms of these studies are the lack of empirical evidence on the effectiveness of educational interventions in clinical reasoning learning (3) and absence of theoretical frameworks in the existing educational interventions (4).

To fill the above-mentioned gaps (lack of empirical evidence and absence of theoretical in the existing educational interventions), some researchers investigated illness script theory and elicited its educational nature. Lubarsky et al identified some teaching strategies based on script theory in guiding reasoning during clinical encounters and proposed strategies for aligning teaching practices with basic principles of script theory in classrooms and clinical settings (4). They acknowledged the lack of evidence to support the value of these strategies and proposed to justify them by empirical evidence. In this study, educational interventions were designed for illness script development in medical students and interns, based on Lubarsky strategies. Furthermore, the intervention aimed at asking both groups to pay more attention to key features during processing case information.

According to illness script theory, the following predictions were made. First, interns, as a result of more clinical experiences, would construct their clinical case representation based on their semi-structured illness scripts. Therefore, the intervention may help them to better recognize the key features and distinguish between differential diagnoses, which leads to more accurate diagnoses. Second, medical students, on the other hand, are not yet proficient enough to deal with a case in a diagnostic focus efficiently and, hence, will have to go through the case information thoroughly. Thus, the intervention may help them to process the case less elaborately and they have to focus more on key features.

\section{Methods}

\section{Participants}

A total of 15 medical students (fourth year) and 12 interns (sixth year) participated in this experimental study. It takes about 7 years (three and half preclinical years and three and half clinical years) to finish medical school in Iran. In this study, the medical students had just started clinical years and had limited hospital experience and their clinical knowledge was mostly based on textbooks and lectures, whereas interns (eg, sixth year medical students) had hospital training under the supervision of senior residents and attending physicians.

\section{Materials}

The material for participants in medical students' group in both pretest and posttest consisted of a booklet containing an instruction about the procedure. There were 2 groups of scenarios of written descriptions of clinical cases (cases in each group were a differential diagnosis of each other), and there was one blank response sheet for writing all key features after reading each case (the first question). The last blank response sheet was provided for writing the common features between the 2 scenarios in each group (second question) and the discriminating features (third question). These case descriptions were about one-page long. The order of the cases was the same for all participants, with different cases in pretest versus posttest.

The material for interns in both pretest and posttest consisted of a booklet containing an instruction about the procedure and a set of 4 clinical reasoning problems (CRP) $(17,18)$. In each problem, a scenario was presented and interns were asked to select the 2 most likely diagnoses to select key features for each diagnosis and to indicate whether these features were positively or negatively predictive. The order of the problems was the same for all participants. Different cases were used in pretest and posttest.

\section{Procedures}

The intervention for both medical students and interns consisted of 3 phases: pretest, training, and posttest. The interventions started with answering pretest questions, which lasted 60 minutes; then, a one-hour lecture was 
presented on clinical diagnostic process and illness scripts.

Following the lecture, the cases were discussed while solving clinical scenarios, and participants were asked to identify the key features of cases (both common and discriminating) and to gather further information based on their activated scripts. Reflection and feedback were used to improve clinical reasoning competency. For interns, 3 strategies were used: thinking aloud, script-based questioning, and test enhanced learning (4). For medical students, not only the same intervention was held but also script-based reading strategy was applied. In the latter strategy, medical students were directed toward relevant sources in the cases and encouraged to read in a manner that promoted acquisition and linking their clinical knowledge into illness scripts. The last phase was a 60minute posttest.

\section{Analysis}

For each participant, the data from 4 cases in both pretest and posttest were combined to obtain the total scores. The protocols (19) were independently assessed by 2 experts and disagreements between experts were resolved by discussion.

\section{Medical students}

Four measures were used to score medical students' protocols. The total key features were investigated by counting the total number of items that were written down in response to the first question, while correct key features were measured by counting the correct items among the total key features. The latter 2 measures were based on the answers to the second and third questions. Common correct key features were the number of correct common key features written in response to question 2. The correct discriminating key features were counted and divided by the total number of key features (eg, correct discriminating key features score). Key features score, common key features score, and discriminative key features score, and total score (The maximum score was 8.) were acquired using the below formulas:

Key features score $=$ Correct key feaures $/$ total key features Common key features score $=$ correct common key features $/$ total common key features
Discriminate key features score $=$ Correct discriminative key features / total discriminative key features

Total score $=$ Key features score + common key features score + discriminate key features score

\section{Interns}

Several clinical reasoning problems were the protocols of the interns. The first and second diagnoses and the selected diagnostic features were scored in each problem. One point was given to each correct diagnosis and each correct key feature. Thus, the maximum score for each problem was 12 points, and the maximum score of pretest or posttest was 48 points. Five measures were used to score the interns' protocols. Total diagnostic accuracy was the sum of the scores of 8 diagnoses that were selected (The maximum score was 8.). Total key feature score was all the correctly selected diagnostic features (Maximum score was 40.). Total CRP score was the sum of the total key feature scores and total diagnostic accuracy (Maximum score was 48.). The total correct discriminating score was the correctly selected diagnostic feature that discriminated the 2 competing diagnoses. The total correct discriminating ratio was measured by total correct discriminating score divided by 5 (the maximum correct discriminating score).

\section{Statistical methods}

SPSS version 21.0 and Stata version 13 were used for statistical analysis. In addition to descriptive statistics, the differences in participants' pretest and posttest variables were tested using Wilcoxon. Significance level was set at $\mathrm{p} \leq 0.05$ for all tests.

\section{Results}

\section{Medical students}

Table 1 shows the Median [ $\left.\mathrm{p}_{25}-\mathrm{p}_{75}\right]$ of the 6 measures. Statistical analysis indicated that educational intervention in medical students had a significant effect on total key features $(p=0.005)$, key feature score $(p=0.001)$, and total score $(\mathrm{p}=0.001)$, but no significant effect on common key features score, discriminating key feature score and correct key features.

\begin{tabular}{|c|c|c|c|}
\hline \multirow[t]{2}{*}{ Variables } & \multicolumn{2}{|c|}{$\operatorname{Median}\left[\mathrm{p}_{25-} \mathrm{p}_{75}\right]$} & \multirow[t]{2}{*}{$\mathrm{p}$} \\
\hline & $\begin{array}{c}\text { Pretest } \\
(\mathrm{N}=15)\end{array}$ & $\begin{array}{l}\text { Posttest } \\
(\mathrm{N}=15)\end{array}$ & \\
\hline Total key features & $85[54-117]$ & $54[44-70]$ & 0.005 \\
\hline Correct key features & $31[24-38]$ & $34[29-40]$ & 0.160 \\
\hline Key feature score & $1.59[1.22-1.86]$ & $2.44[2.22-2.87]$ & 0.001 \\
\hline Common key feature score & $0.4[0.25-78]$ & $0.9[0.6-1]$ & 0.791 \\
\hline Discriminating key feature score & $0.33[0.16-0.33]$ & $0.22[0.11-.44]$ & 0.972 \\
\hline Total score & $2.28[2.10-2.57]$ & $3.69[3.35-4.93]$ & 0.001 \\
\hline \multicolumn{4}{|c|}{ Table 2. Comparisons of pretest and posttest scores in interns } \\
\hline \multirow[t]{2}{*}{ Variables } & \multicolumn{2}{|c|}{ Mean (SD) } & $\mathrm{p}$ \\
\hline & $\begin{array}{l}\text { Pretest } \\
\mathrm{N}=12\end{array}$ & $\begin{array}{l}\text { Posttest } \\
\mathrm{N}=12\end{array}$ & \\
\hline Total diagnostic accuracy & $5.41(1.16)$ & $4.91(1.44)$ & 0.111 \\
\hline Total key features score & $13.53(6.77)$ & $18.08(4.75)$ & 0.005 \\
\hline Total CRP score & $18.75(8.05)$ & $24.08(8.05)$ & 0.001 \\
\hline Total correct discriminating score & $0.41(0.66)$ & $1.41(2.06)$ & 0.146 \\
\hline
\end{tabular}




\section{Interns}

Table 2 demonstrates the mean and standard deviations of the 4 measures. Statistical analysis indicated that educational intervention for interns had a significant effect on total key features score $(\mathrm{p}=0.005)$ and total CRP score $(\mathrm{p}=0.001)$.

\section{Discussion}

Clinical reasoning is a mental process that plays an important role in the accurate diagnosis and management of diseases $(20,21)$. The aim of this study was to investigate the impact of educational strategies inspired by script theory in the development of illness scripts. It was expected that interns construct their clinical case representation based on their semi-structured illness scripts because they had more clinical experiences. Therefore, the intervention may help them to better recognize common and discriminating features and to make more accurate diagnoses. On the other hand, it was predicted that medical students will not yet be proficient enough to deal with the case in a diagnostic focus efficiently and, hence, should go through the case information very carefully. Thus, the intervention may help them to process the case less elaborately and to focus on key features more deliberately. The fact that interns' CRP average score at the highest level was half of the total score may indicate that they were not a good representative of their level of expertise. Moreover, previous studies that assessed clinical reasoning in interns with CRP showed the same results $(18,22,23)$.

The results of previous studies were in line with our assumptions in that the interns recognized considerably more key features in the posttest. However, there was inconsiderable difference between pretest and posttest in total diagnostic accuracy and total correct discriminating score, which is not in line with our predictions. It seems that the interns were more curious about the common key features of diseases rather than paying attention to discriminating key features and being concerned about differentiating the competing diagnoses that led to no change in diagnostic accuracy. It seems that these findings were supported by some previous studies in which final diagnostic accuracy and differential diagnosis accuracy were insensitive to short-term educational interventions (24, 25).

Findings were also partially in line with our predictions in that medical students produced less total key features in the posttest and the increase in correct key features, indicating that they become less elaborate in their case processing. The insignificant difference in correct key features score, common key feature score, and discriminative key features score in posttest compared to pretest may demonstrate that medical students tend to choose key features rather than being accurate. These findings are in line with previous studies that indicated medical students are relatively insensitive to the nature and circumstances of the task (26-33).

Translation of findings from such basic research to applications in medical education is not so trivial. Showing the way medical students and interns deal with written cases in a crash course does not directly guide us on how to train them in real clinical settings. However, some recommendations can still be made for medical education. This study showed that such theory-based studies can bring about fresh and insightful ideas for teaching clinical reasoning and may fill in the gap between theory and practice in medical education research. It seems that script-based learning may lead to a change in the weighing of features, a change in the number of features learned, or a new relationship being encoded among features, thus, further research is needed to investigate these issues.

\section{Conclusion}

This study showed that theory-driven educational strategies have an impact on illness script development- specifically in interns. It seems that this intervention would be tested on those in higher levels of expertise (ie, residents). It was difficult to estimate the long-term effect of this educational intervention, as the testing phase took place immediately after the learning phase. Also, this study recommends that this intervention be used in real clinical settings.

\section{Acknowledgments}

Authors would like to acknowledge Iran University of Medical Sciences for providing financial support. Extend thanks to medical students and interns for their participation in the study.

\section{Funding}

The present study was financially supported by Iran University of Medical Sciences, for partial fulfillment of Ph.D. dissertation, (Grant No: 6784). The funding organization did not play any roles in the study design, collection, analysis, and interpretation of data, or in writing the manuscript.

\section{Ethics approval and consent to participate}

This study was approved by the Ethical Review Board of Iran University of Medical Sciences (approval number: IR.IUMS.REC1395.9321486001). Informed consent was obtained from all participants. Participation in this study was voluntary and codes were given to students to ensure their anonymity and to ensure that the study had no effects on their mandatory final exams. Also, faculty members were assured that the results of the study and test scores will remain confidential and will only be released as a group.

\section{Conflict of Interests}

The authors declare that they have no competing interests.

\section{References}

1. Harasym PH, Tsai TC, Hemmati P. Current trends in developing medical students' critical thinking abilities. Kaohsiung J Med Sci. 2008;24(7):341-55.

2. Charlin B, Tardif J, Boshuizen HP. Scripts and medical diagnostic knowledge: theory and applications for clinical reasoning instruction and research. Acad Med. 2000;75(2):182-90.

3. Schmidt HG, Mamede S. How to improve the teaching of clinical reasoning: a narrative review and a proposal. Med Educ. 
2015;49(10):961-73.

4. Lubarsky S, Dory V, Audétat MC, Custers E, Charlin B. Using script theory to cultivate illness script formation and clinical reasoning in health professions education. Can Med Educ J. 2015;6(2):e61.

5. Keemink Y, Custers EJ, van Dijk S, ten Cate O. Illness script development in pre-clinical education through case-based clinical reasoning training. Int J Med Educ. 2018;9:35.

6. Schmidt HG, Rikers RM. How expertise develops in medicine: knowledge encapsulation and illness script formation. Med Educ. 2007;41(12):1133-9.

7. Schmidt H, Norman G, Boshuizen HP. A cognitive perspective on medical expertise: theory and implications. Acad Med. 1990;65(10):611-21.

8. Custers EJFM. Thirty years of illness scripts: theoretical origins and practical applications. Med Teach. 2015;37(5):457-62.

9. Custers EJ, Boshuizen HP, Schmidt HG. The role of illness scripts in the development of medical diagnostic expertise: Results from an interview study. Cogn Instr. 1998;16(4):367-98.

10. Schmidt HG, Boshuizen HP. On acquiring expertise in medicine. Educ Psychol Rev.1993;5(3):205-21.

11. Monajemi A, Rostami ER, Savaj S, Rikers RM. How does patient management knowledge integrate into an illness script? Educ Health (Abingdon). 2012;25(3):153-9.

12. Strasser J, Gruber H. Learning processes in the professional development of mental health counselors: knowledge restructuring and illness script formation. Adv Health Sci Educ. 2015;20(2):51530 .

13. Sadeghi, Asgari AA, Moulaei N, Mohammadkarimi V, Delavari S, Amini M, et al. Combination of different clinical reasoning tests in a national exam. J Adv Med Educ Prof. 2019;7(7):227-31.

14. Woods NN. Science is fundamental: the role of biomedical knowledge in clinical reasoning. Med Educ. 2007;41(12):1173-7.

15. Schmidt HG, Rikers RM. How expertise develops in medicine: knowledge encapsulation and illness script formation. Med Educ. 2007;41(12):1133-9.

16. Charlin B, Boshuizen H, Custers EJ, Feltovich PJ. Scripts and clinical reasoning. Med Educ. 2007;41(12):1178-84.

17. ten Cate O, Durning SJ. Approaches to Assessing the Clinical Reasoning of Preclinical Students. Principles and Practice of Casebased Clinical Reasoning Education: Springer; 2018. p. 65-72.

18. Groves M, Dick ML, McColl G, Bilszta J. Analysing clinical reasoning characteristics using a combined methods approach. BMC Med Educ. 2013;13(1):144.

19. Boshuizen HP, Schmidt HG. On the role of biomedical knowledge in clinical reasoning by experts, intermediates and novices. Cogn Sci. 1992;16(2):153-84

20. Delavari S, Amini M, Sohrabi Z, Koohestani H, Delavari S, Rezaee $\mathrm{R}$, et al. Development and psychometrics of script concordance test (SCT) in midwifery. Med J Islam Repub Iran. 2018;32:75.

21. Zamani S, Amini M, Masoumi SZ, Delavari S, Namaki MJ, Kojuri J. The comparison of the key feature of clinical reasoning and multiple choice examinations in clinical decision makings ability. Biomed Res India. 2017;28(3).

22. Derakhshandeh Z, Amini M, Kojuri J, Dehbozorgian M. Psychometric characteristics of clinical reasoning problems (CRPs) and its correlation with routine multiple choice question (MCQ) in cardiology department. J Adv Med Educ Prof. 2018;6(1):37.

23. Amini M, Moghadami M, Kojuri J, Abbasi H, Abadi AAD, Molaee NA, et al. An innovative method to assess clinical reasoning skills: Clinical reasoning tests in the second national medical science Olympiad in Iran. BMC Res Notes. 2011;4(1):418.

24. Littlefield JH, Hahn HB, Meyer AS. Evaluation of a role-play learning exercise in an ambulatory clinic setting. Adv Health Sci Educ. 1999;4(2):167-73.

25. Montaldo G, Herskovic P. Teaching of clinical reasoning to medical students using prototypical clinical cases. Rev Med Chil. 2013;141(7):823-30.

26. de Bruin AB, Schmidt HG, Rikers RM. The role of basic science knowledge and clinical knowledge in diagnostic reasoning: a structural equation modeling approach. Acad Med. 2005;80(8):76573.

27. Rikers RM, Schmidt HG, Boshuizen HP. Knowledge encapsulation and the intermediate effect. Contemp Educ Psychol. 2000;25(2):15066.

28. Rikers RM, Schmidt HG, Boshuizen HP. On the constraints of encapsulated knowledge: Clinical case representations by medical experts and subexperts. Cogn Instr. 2002;20(1):27-45.

29. Rikers RM, Loyens SM, Schmidt HG. The role of encapsulated knowledge in clinical case representations of medical students and family doctors. Med Educ. 2004;38(10):1035-43.

30. Monajemi A, Rikers RM, Schmidt HG. Clinical case processing: a diagnostic versus a management focus. Med Educ. 2007;41(12):116672.

31. Monajemi A, Schmidt HG, Rikers RM. Assessing patient management plans of doctors and medical students: An illness script perspective. J Contin Educ Health Prof. 2012;32(1):4-9.

32. Rikers RM, Schmidt HG, Boshuizen H, Linssen G, Wesseling G, Paas FG. The robustness of medical expertise: Clinical case processing by medical experts and subexperts. Am J Psychol. 2002; 115(4):609-29.

33. Verkoeijen $\mathrm{P}$, Imbos $\mathrm{T}$, van de Wiel $\mathrm{M}$, Berger M, Schmidt $\mathrm{H}$. Assessing knowledge structures in a constructive statistical learning environment. J Stat Educ. 2002;10(2). 